\title{
Inhibition of 2-Arachidonoylglycerol Metabolism Alleviates Neuropathology and Improves Cognitive Function in a Tau Mouse Model of Alzheimer's Disease
}

\section{Jack Hashem}

UT Health San Antonio: The University of Texas Health Science Center at San Antonio

Mei Hu

UT Health San Antonio: The University of Texas Health Science Center at San Antonio Jian Zhang

UT Health San Antonio: The University of Texas Health Science Center at San Antonio Fei Gao

UT Health San Antonio: The University of Texas Health Science Center at San Antonio Chu Chen ( $\square$ chenc7@uthscsa.edu )

UT Health San Antonio Long School of Medicine: The University of Texas Health Science Center at San Antonio Joe R and Teresa Lozano Long School of Medicine https://orcid.org/0000-0003-1287-8059

\section{Research Article}

Keywords: Endocannabinoid, 2-arachidonoylglycerol, monoacylglycerol lipase, Alzheimer's disease, neuroinflammation, neuropathology, cognition

Posted Date: March 10th, 2021

DOl: https://doi.org/10.21203/rs.3.rs-271559/v1

License: (a) (1) This work is licensed under a Creative Commons Attribution 4.0 International License. Read Full License 


\section{Abstract}

Alzheimer's disease (AD) is the most common cause of dementia, which affects more than 5 million individuals in the United States. Unfortunately, no effective therapies are currently available to prevent development of $A D$ or to halt progression of the disease. It has been proposed that monoacylglycerol lipase (MAGL), the key enzyme degrading the endocannabinoid 2-arachidonoylglycerol (2-AG) in the brain, is a therapeutic target for AD based on the studies using the APP transgenic models of AD. While inhibition of 2-AG metabolism mitigates b-amyloid $(A \beta)$ neuropathology, it is still not clear whether inactivation of MAGL alleviates tauopathies as accumulation and deposition of intracellular hyperphosphorylated tau protein are the neuropathological hallmark of AD. Here we show that JZL184, a potent MAGL inhibitor, significantly reduced proinflammatory cytokines, astrogliosis, phosphorylated tau, and GSK3b in P301S/PS19 mice, a tau mouse model of AD. Importantly, Tau transgenic mice treated with JZL184 displayed improvements in spatial learning and memory retention. In addition, inactivation of MAGL ameliorates deteriorations in expression of synaptic proteins in P301S/PS19 mice. Our results provide further evidence that MAGL is a promising therapeutic target for AD.

\section{Introduction}

Alzheimer's disease (AD), the most common form of dementia, is a degenerative brain disease characterized by neuroinflammation, accumulation of $\beta$-amyloid (A $\beta)$ plaques and neurofibrillary tangles, neurodegeneration, synaptic dysfunction, and cognitive decline. Based on 'the 2020 Alzheimer's Disease Facts and Figures' reported by Alzheimer's Association, more than 5 million Americans age 65 and older are living with Alzheimer's dementia in 2020 and AD is the sixth-leading cause of death in the US. However, no effective pharmacotherapies are currently available for prevention and treatment of $A D$. This is largely due to the fact that etiology of $A D$ is multifactorial and development of $A D$ is linked to multiple mechanisms or signaling pathways. This means that the ideal $A D$ therapy should be able to modify the disease through multiple mechanisms.

Endocannabinoids are endogenous bioactive lipid mediators involved in a variety of physiological and pathological processes. The endocannabinoid 2-arachidonoylglycerol (2-AG) is the most abundant endogenous cannabinoid and a full agonist for both CB1R and CB2R [1]. Several lines of evidence indicate that 2-AG displays anti-inflammatory and neuroprotective properties in responses to harmful insults [2-6]. 2-AG is primarily synthesized from diacylglycerol (DAG) by diacylglycerol lipases $\alpha / \beta$ and metabolized by the enzymes, including monoacylglycerol lipase (MAGL) and $\alpha / \beta$ hydrolase domaincontaining protein 6 and 12 (ABHD6/12). 2-AG is also converted by cyclooxygenase-2 (COX-2) to a new class of prostaglandins, prostaglandin glycerol esters (PG-Gs), when COX-2 is excessively activated during inflammation [6, 7]. Although these enzymes are capable of metabolizing of 2-AG, it has been estimated that $85 \%$ of $2-A G$ in the brain is degraded by MAGL $[8,9]$, suggesting that MAGL plays a dominant role in control of endogenous 2-AG levels in the brain. Recently studies provided further evidence that 2-AG in neurons and astrocytes is primarily hydrolyzed by MAGL, while it in microglial cells is predominantly degraded by ABHD12 $[10,11]$. The immediate metabolites of 2-AG are arachidonic acid 
(AA), a precursor of prostaglandins catalyzed by COX-1/2 and leukotrienes via arachidonate 5lipoxygenase (LOX). Prostaglandins (i.e., PGE2) and leukotrienes are important inflammatory mediators or proinflammatory $[12,13]$, while 2-AG is anti-inflammatory and neuroprotective [2-6, 14]. Apparently, inactivation of MAGL would result in augmentation of anti-inflammatory and neuroprotective 2-AG signaling, while lowering proinflammatory and neurotoxic eicosanoid levels $[6,7,15]$, suggesting that inhibition of 2-AG metabolism in the brain will lead to activate neuroprotective signaling pathways and concurrently turn off signaling pathways that are detrimental to brain function. Indeed, previous studies from our group and others demonstrated that inactivation of MAGL reduces $A \beta$ formation and accumulation, neuroinflammation and neurodegeneration and improves synaptic and cognitive functions in APP transgenic mice, animal models of AD [16-19]. However, it is still not clear whether inhibition of 2AG metabolism mitigates tauopathies as intracellular neurofibrillary tangles that represent hyperphosphorylated tau proteins are an important neuropathological hallmark of $A D[20,21]$. Here we show that inactivation of MAGL attenuates neuroinflammation and tau phosphorylation and prevents deterioration in synaptic proteins and cognitive decline in P301S/PS19 mice, a tau mouse model of AD, supporting generalizability of MAGL as a promising therapeutic target for AD.

\section{Results}

\section{JZL184 mitigates neuroinflammation in P301S/PS19 mice}

Neuroinflammation is one of the major pathogenic mechanisms that contribute to neuropathology and cognitive decline in AD. Previous studies demonstrate that pharmacological inhibition MAGL attenuates neuroinflammation evident by decreases in proinflammatory cytokines and astroglial reactivity in APP transgenic mice [16-19]. To determine whether inhibition of MAGL produces anti-inflammatory effects in tau P301S/PS19 transgenic (TG) mice [22], we treated mice at 5 months of age with JZL184 $(10 \mathrm{mg} / \mathrm{kg}$, three times/week for 8 weeks, Fig. 1A), as described previously [16]. We first assessed hippocampal cytokines IL-1 $\beta$ and TNFa in 7-month old TG mice and age-matched wild type (WT) control mice treated with vehicle or JZL184 using ELISA analysis. As shown in Fig. 1B, the levels of IL-1 $\beta$ and TNFa were significantly elevated in PS19 TG mice when compared with WT mice. Treatment with JZI184 significantly decreased hippocampal IL-1 $\beta$ and TNFa in both WT and TG mice. These results indicate that inhibition of 2-AG metabolism with JZL184 reduces neuroinflammation in tau TG mice. To further confirm anti-inflammatory effects of MAGL inactivation in tau TG mice, GFAP immunoreactivity, a marker for inflammatory responses in astrocytes, was assessed in the brain of PS19 TG mice treated with vehicle or JZL184 three times a week for 8 weeks. As shown in Fig. 2, GFAP immunoreactivity was robustly reduced in the cortex and hippocampus of 7-month old PS19 TG mice treated with JZL184. Next, we detected Iba1 immunoreactivity, a marker for inflammatory responses in microglia, as microglial cells are the innate immune system of the brain and play an important role in neuroinflammatory processes. As shown in Fig. 3, JZL184 also greatly suppressed reactive microglial cells in the cortex and hippocampus of the PS19 TG mice. These results are consistent with previous observations where inhibition of MAGL mitigates neuroinflammation in APP TG mice [16-18]. 


\section{Inhibition of 2-AG metabolism alleviates tauopathies in P301S/PS19 TG mice}

Accumulation of intracellular neurofibrillary tangles resulting from hyperphosphorylated tau is the neuropathological hallmark of $A D[20,21]$. However, no studies have been conducted to assess whether disruption of 2-AG metabolism reduces phosphorylated tau ( $\mathrm{p}$-tau). To this end, we assessed total tau (Tau-5) and p-tau, including p-tau Thr181 (p-tauT181), and p-tau Ser202/Thr205 (AT8) in the hippocampus of PS19 TG mice treated with JZL184. As shown in Fig. 4, p-tauT181 and AT8 were robustly elevated in TG mice, indicating increased p-tau proteins in PS19 TG mice. Importantly, while JZL184 did not induce changes in Tau-5, which is the transgene and overexpressed in this line of tau TG mice, it significantly reduced p-tauT181 and AT8 in TG mice, indicating that inactivation of MAGL decreases $p$-tau. Since tau phosphorylation is largely regulated by glycogen synthase kinase-3 $\beta$ (GSK3 $\beta$ ) and cyclin-dependent kinase 5 (Cdk5) [23-26], we detected phosphorylated GSK3 $\beta$ (p-GSK3 $\beta$ ) and P35/25 that interact with Cdk5. As shown in Fig. 4, p-GSK3 $\beta$ and P25 were significantly elevated in TG mice that received the vehicle, but the increase was reduced in TG mice treated JZL184, indicating JZL184-reduced $p$-tau is likely through suppression of p-GSK3 $\beta$ and P25.

\section{Inactivation of MAGL improves cognitive function in P301S/PS19 TG mice}

Inactivation of MAGL has been shown to improve spatial learning and memory in APP transgenic mice $[16,19,27]$. However, it is still not clear whether inactivation of MAGL prevents cognitive decline in tau transgenic models of AD. To determine whether inhibition of 2-AG metabolism improves cognitive function, we assessed spatial learning and memory retention using the novel object recognition (NOR) and the Morris water maze tests, as described previously [16, 19, 27-30], in PS19 TG mice treated with JZL184 for 8 weeks. As shown in Fig. 5A, memory retention was significantly impaired in 7-month-old PS19 TG mice treated with vehicle. However, the impairment was prevented in TG mice treated with JZL184. The cognitive improvement by inactivation of MAGL in PS19 TG mice is further confirmed by the Morris water maze test. As shown in Fig. 5B D, JZL184 significantly improves spatial learning and memory retention in PS19 TG mice, similar to APP transgenic mice $[16,19,27]$. These results suggest that inactivation of MAGL is capable of preventing cognitive decline in both APP and tau transgenic models of $A D$.

\section{Inhibition of 2-AG metabolism prevents deterioration in expression of synaptic proteins in P301S/PS19 TG mice}

It has been well recognized that cognitive deficits in $A D$ is primarily due to loss of the synaptic integrity $[29,31]$. Proper and efficient synaptic transmission and plasticity in the brain are largely dependent on expression and function of excitatory glutamate receptors. Our previous studies showed that inactivation of MAGL in APP TG mice prevents downregulation of glutamate receptor subunits in the brain [16, 27]. To 
determine whether JZL184 prevents deterioration in expression of glutamate receptors in tau TG mice, we detected expression of AMPA and NMDA glutamate receptor subunits, including GluA1, GluA2, GluN1, GluN2A and GluN2B, in PS19 mice treated with vehicle or JZL184. As shown in Fig. 6, expression of GluA1, GluA2, GluN2A, and GluN2B in the hippocampus was significantly downregulated in PS19 TG mice treated with vehicle. However, expression of these glutamate receptor subunits in mice treated with JZL184 was returned to the levels in WT mice, suggesting that inhibition of 2-AG metabolism is also capable of preventing deterioration in expression of glutamate receptor subunits. To further assess whether inactivation of MAGL prevents changes in expression of synaptic proteins in PS19 TG mice, we also detected synaptophysin (Syn) and PSD-95, pre- and post-synaptic markers, and PPARy, an important nuclear receptors displaying anti-inflammatory and neuroprotective effects $[19,32,33]$. We observed that hippocampal expression of Syn, PSD-95, and PPARy was significantly reduced in 7-months old PS19 TG mice. However, treatment with JZL184 attenuated downregulation of Syn and PPARY. Although expression of PSD-95 in JZL184-treated animals was slightly increased, but no statistical significance. Our results provide evidence that restraint of 2-AG metabolism by inactivation of MAGL averts downregulation of synaptic proteins in tau TG mice.

\section{Discussion}

It has been proposed that MAGL inhibition, which augments 2-AG signaling and reduces eicosanoid levels [8, 9], is a promising therapy for AD [16]. However, MAGL proposed as a therapeutic target is largely based on the studies using the APP transgenic models of AD [16-19, 27]. Since intracellular neurofibrillary tangles resulting from accumulation of hyperphosphorylated tau proteins is one of the important neuropathological hallmarks of $A D$, it is still not clear whether inhibition of 2-AG metabolism alleviates tauopathies. To ascertain generalizability of the results from APP TG mice, we used P301S/PS19 TG mice, a tau mouse model of AD [22]. We demonstrate in the present study that JZL184, a highly selective and potent MAGL inhibitor $[9,34,35]$, significantly reduces neuroinflammation and phosphorylated tau, prevents deteriorations in expression of synaptic proteins, and improves cognitive function in P301S/PS19 mice. Our results provide further evidence that inactivation of MAGL is capable of alleviating both $A \beta$ neuropathology and tauopathies, supporting the notion that MAGL is a promising therapeutic target for $A D$.

It is generally accepted that the etiology of $A D$ is multifactorial and complex. Apparently, $A D$ involves multiple etiopathogenic mechanisms or signaling pathways. This means that the ideal AD therapy should be able to modify the disease through multiple mechanisms. Several lines of evidence suggest that 2-AG is an important endogenous signaling molecule maintaining brain homeostasis against harmful insults via its anti-inflammatory and neuroprotective properties [2-6, 14, 36, 37], while the metabolites of 2-AG are proinflammatory and neurotoxic $[12,13]$. Therefore, inactivation of MAGL, which augments 2-AG signaling and reduces its metabolites, turns on anti-inflammatory and neuroprotective signaling pathways and concurrently off signaling pathways that are detrimental to homeostatic maintenance of brain function [15]. Previous studies provide evidence showing that pharmacological or genetic inactivation of MAGL produces profound anti-inflammatory and neuroprotective effects and improves 
synaptic and cognitive functions in animal models of AD and MPTP model of Parkinson's disease [9, 1618]. These beneficial effects of MAGL inhibition are likely mediated through multiple signaling pathways.

Senile plaques and neurofibrillary tangles are the two neuropathological hallmarks of AD. Senile plaques are extracellular accumulation and deposition of $A \beta$, while neurofibrillary tangles are intracellular deposition of hyperphosphorylated tau proteins. The behavioral symptoms of $A D$ are largely associated with the accumulation of plaques and tangles [20]. Although both $A \beta$ and $p$-tau contribute to neuropathology, synaptic and cognitive declines in $A D$, accumulated evidence suggests that tau proteins are essential or required for $A \beta$-induced neurotoxicity, neurodegeneration and synaptic dysfunction [21, $38,39]$. This means that hyperphosphorylated tau plays an important role in driving cognitive decline in AD. In addition, clinical trials of $A \beta$-targeting therapies are largely failed. This suggests that efficacious $A D$ therapies may need to alleviate both $A \beta$ and tau pathologies. The results from the previous studies where inactivation of MAGL alleviates neuropathology and improves synaptic and cognitive function in APP TG mice [16-19,27] together with the findings from the present study in tau TG mice suggest that inhibition of 2-AG metabolism is capable of curbing both $A \beta$ and tau neuropathologies and preventing cognitive decline and that MAGL is a promising therapeutic target.

\section{Materials And Methods}

\section{Animals}

P301S(PS19) transgenic mice (B6;C3-Tg(Prnp-MAPT*P301S)PS19Vle/J, stock number: 008169) were obtained from the Jackson Lab [22]. Both male and female PS19 mice and age-matched non-transgenic wild-type (WT) littermates at 7 months of age were used in the present study. 4-Nitrophenyl-4-[bis (1,3benzodioxol-5-yl)(hydroxy)methyl]piperidine-1-carboxylate (JZL184, provided by the NIH Mental Health Institute Chemical Synthesis and Drug Supply Program), a potent and selective inhibitor of MAGL, was dissolved in the vehicle containing Tween-80 (10\%), DMSO (10\%), and saline (80\%). Animals were treated with either vehicle or JZL184 (10 mg/kg, i.p.) three times per week starting at 5 months of age for 8 weeks, as described previously $[16,19]$. All the observations and assessments in mice were made at 7 months of age.

All the experiments were performed in compliance with the US Department of Health and Human Services Guide for the Care and Use of Laboratory Animals.

The care and use of the animals reported in this study were approved by the Institutional Animal Care and Use Committee of University of Texas Health San Antonio.

The experiments, whenever possible, were performed in a blinded fashion.

\section{ELISA.}

Levels of IL-1 $\beta$ and TNFa in hippocampal tissues of PS19 TG mice that received vehicle control or JZL184 were detected using a colorimetric IL1 $\beta$ and TNFa ELISA kits (IL-1 $\beta$ : Cat\# RAB0275, Sigma- 
Aldrich, and TNFa: Cat\# MBS825075, MyBioSource) according to the instructions provided by the manufacturers.

\section{Immunoblot}

Western blot assay was conducted to determine expression of GluA1, GluA2, GluN1, GluN2A, and GluN2B, tau-5, AT8, p-tauT181, p-GSK3 $\beta$, p35/25, PPARy, PSD95, synaptophysin (Syn) in the hippocampus from animals that received vehicle or JZL184. Hippocampal tissue was extracted and immediately homogenized in RIPA lysis buffer and protease inhibitors, and incubated on ice for $30 \mathrm{~min}$, then centrifuged for $10 \mathrm{~min}$ at $10,000 \mathrm{rpm}$ at $4^{\circ} \mathrm{C}$. Supernatants were fractionated on 4-15\% SDS-PAGE gels and transferred onto PVDF membranes (Bio-Rad). The membrane was incubated with anti-GluA1 (1:1,000, Abcam, Cat\# AB31232), GluA2 (1:1,000, Abcam, Cat\# AB133477), GluN1 (1:1,000, Abcam, Cat\# AB109182), GluN2A (1:1,000, LSBio, Cat\# LS-B7707), GluN2B (1:1,000, Abcam, Cat\# AB65783), tau-5 (1:1,000, ThermoFisher, Cat\# AHB0042), AT8 (1:200, ThermoFisher, Cat\# MN1020), p-tauT181 (1:1,000, Cell Signaling Technology, Cat\# 12885), p-GSK3 3 (1:1,000, Cell Signaling Technology, Cat\# 9323), p35/25 (1:1,000, Cell Signaling Technology, Cat\# 2680), PPARy (1:1,000, Abcam, Cat\# AB27649), Syn (1:500, Abcam, Cat\# AB8049), and PSD95 (1:1,000, Abcam, Cat\# AB2723) at $4^{\circ} \mathrm{C}$ overnight. The blots were washed and incubated with a secondary antibody (goat anti-rabbit 1:2,000, Cell Signaling Technology, Cat\# 7074) at room temperature for $1 \mathrm{hr}$. Proteins were visualized by enhanced chemiluminescence (Amersham Biosciences, UK). The densities of specific bands were quantified by densitometry using FUJIFILM Multi Gauge software (version 3.0). Band densities were quantified and converted to the total amount of protein loaded in each well as determined by mouse anti $\beta$-actin (1:2,000, Santa Cruz Biotechnology, Cat\# SC-47778) as described previously $[16,19,28,29]$.

\section{Immunohistochemistry}

Immunohistochemical analyses were performed to determine astrocytic and microglial markers (GFAP and Iba1) in coronal brain sections, as described previously $[16,19,30]$. Animals were anesthetized with ketamine/Xylazine $(200 / 10 \mathrm{mg} / \mathrm{kg}$ ) and subsequently transcardially perfused with PBS followed by $4 \%$ paraformaldehyde in phosphate buffer. The brains were quickly removed from the skulls and fixed in $4 \%$ paraformaldehyde overnight, and then transferred into the PBS containing $30 \%$ sucrose until sinking to the bottom of the small glass jars. Cryostat sectioning was made on a freezing Vibratome at $40 \mu \mathrm{m}$ and series sections (every 10 th section from each animals) were collected in $0.1 \mathrm{M}$ phosphate buffer. Free floating sections were immunostained using specific antibodies for GFAP (1:500, Sigma-Aldrich, Cat\# G3893) an Iba1 (1:500, Sigma-Aldrich, Cat\# MABN92) followed by incubation with the corresponding fluorescent-labeled secondary antibody. 4'-6-Diamidino-2-phenylindole (DAPI), a fluorescent stain that binds strongly to DNA, was used it to detect cell nuclei in the sections. The sections were then mounted on slides for immunofluorescence detection using a Zeiss deconvolution microscope. The immunoreactivity (in arbitrary densitometric units) of GFAP and lba1 in the cortex and hippocampus in each image was analyzed and quantified using SlideBook 6.0, as described previously $[16,19,27,30]$.

\section{Novel object recognition}


The novel object recognition (NOR) test was performed as described previously [29]. Briefly, animals were first allowed to acclimate to the testing environment (habituation). The test included two stages: training and testing. In the first stage of the test, the animal was confronted with two identical objects, placed in an open field, and in the second stage, the animal was exposed to two dissimilar objects placed in the same open field: one familiar object, used in the first phase, and the other novel object. Exploration of an object was defined as time spent with the head oriented towards and within two $\mathrm{cm}$ of the object. The time spent exploring each of the objects in stage two was detected by a video-camera using an EthoVision video tracking system (Noldus).

\section{Morris water Maze}

The classic Morris water maze (MWM) test was used to determine spatial learning and memory, as described previously $[16,19,27-30]$. Animals were initially randomized grouped to receive different treatments. The test was performed 24 hrs after last cessation of JZL184 injections. A circular water tank (diameter $120 \mathrm{~cm}$ ) was filled with water and the water was made opaque with non-toxic white paint. A round platform (diameter $15 \mathrm{~cm}$ ) was hidden $1 \mathrm{~cm}$ beneath the surface of the water at the center of a given quadrant of the water tank. PS19 TG mice and their age-matched littermates received learning acquisition training for 7 days ( 7 sessions), and each session consisted of 4 trials. For each trial, the mouse was released from the wall of the tank and allowed to search, find, and stand on the platform for 10 seconds within the 60-second trial period. For each training session, the starting quadrant and sequence of the four quadrants from where the mouse was released into the water tank were randomly chosen so that it was different among the separate sessions for each animal and was different for individual animals. The mice in the water pool were recorded by a video-camera using an EthoVision video tracking system (Noldus). A probe test to assess memory retention was conducted 24 hours after the completion of the training acquisition. During the probe test, the platform was removed from the pool, and the task performances were recorded for 60 seconds.

\section{Data analysis}

Data are presented as mean \pm S.E.M. Unless stated otherwise, one- or two-way-analysis of variance (ANOVA) followed by post-hoc tests were used for statistical comparison when appropriate. Differences were considered significant when $\mathrm{P}<0.05$.

\section{Declarations}

\section{Acknowledgement}

The authors also thank NIH Mental Health Institute Chemical Synthesis and Drug Supply Program for providing JZL184.

\section{Author Contributions}


C.C. conceived the project and designed the experiments; J.H., M.H., J.Z., F.G. and C.C. performed the experiments and analyzed the data; C.C. supervised the work and wrote the manuscript.

\section{Funding}

This work was supported by National Institutes of Health grants R01NS076815, R01MH113535, and R01AG058621 (to C.C.) and by startup funds from UT Health San Antonio, Joe R. \& Teresa Lozano Long School of Medicine (to C.C.).

\section{Conflict of interest}

The authors declare no conflict of interest.

\section{Ethics Approval}

All the animal experiments were performed in compliance with the US Department of Health and Human Services Guide for the Care and Use of Laboratory Animals. The care and use of the animals reported in this study were approved by the Institutional Animal Care and Use Committee of University of Texas Health San Antonio.

\section{Data availability}

Data supporting the findings of this manuscript are available from the corresponding authors upon request.

\section{Code availability}

Not applicable

\section{Consent to participate}

Not applicable

\section{Consent for publication}

Not applicable

\section{References}

1. Sugiura T, Kishimoto S, Oka S, Gokoh M (2006) Biochemistry, pharmacology and physiology of 2arachidonoylglycerol, an endogenous cannabinoid receptor ligand. Prog Lipid Res 45:405-446. doi:10.1016/j.plipres.2006.03.003

2. Chen X, Zhang J, Chen C (2011) Endocannabinoid 2-arachidonoylglycerol protects neurons against beta-amyloid insults. Neuroscience 178:159-168. doi:10.1016/j.neuroscience.2011.01.024 
3. Du H, Chen X, Zhang J, Chen C (2011) Inhibition of COX-2 expression by endocannabinoid 2arachidonoylglycerol is mediated via PPAR-gamma. $\mathrm{Br} \mathrm{J}$ Pharmacol 163:1533-1549. doi:10.1111/j.1476-5381.2011.01444.x

4. Zhang J, Chen C (2008) Endocannabinoid 2-arachidonoylglycerol protects neurons by limiting COX-2 elevation. J Biol Chem 283:22601-22611. doi:10.1074/jbc.M800524200

5. Panikashvili D, Simeonidou C, Ben-Shabat S, Hanus L, Breuer A, Mechoulam R, Shohami E (2001) An endogenous cannabinoid (2-AG) is neuroprotective after brain injury. Nature 413:527-531. doi:10.1038/35097089

6. Xu JY, Chen C (2015) Endocannabinoids in Synaptic Plasticity and Neuroprotection. Neuroscientist 21:152-168. doi:10.1177/1073858414524632

7. Hermanson DJ, Gamble-George JC, Marnett LJ, Patel S (2014) Substrate-selective COX-2 inhibition as a novel strategy for therapeutic endocannabinoid augmentation. Trends Pharmacol Sci 35:358367. doi:10.1016/j.tips.2014.04.006

8. Blankman JL, Simon GM, Cravatt BF (2007) A comprehensive profile of brain enzymes that hydrolyze the endocannabinoid 2-arachidonoylglycerol. Chem Biol 14:1347-1356. doi:10.1016/j.chembiol.2007.11.006

9. Nomura DK, Morrison BE, Blankman JL, Long JZ, Kinsey SG, Marcondes MC, Ward AM, Hahn YK, Lichtman AH, Conti B, Cravatt BF (2011) Endocannabinoid hydrolysis generates brain prostaglandins that promote neuroinflammation. Science 334:809-813. doi:10.1126/science.1209200

10. Viader A, Blankman JL, Zhong P, Liu X, Schlosburg JE, Joslyn CM, Liu QS, Tomarchio AJ, Lichtman AH, Selley DE, Sim-Selley LJ, Cravatt BF (2015) Metabolic Interplay between Astrocytes and Neurons Regulates Endocannabinoid Action. Cell Rep 12:798-808. doi:10.1016/j.celrep.2015.06.075

11. Viader A, Ogasawara D, Joslyn CM, Sanchez-Alavez M, Mori S, Nguyen W, Conti B, Cravatt BF (2016) A chemical proteomic atlas of brain serine hydrolases identifies cell type-specific pathways regulating neuroinflammation. Elife 5:e12345. doi:10.7554/eLife.12345

12. Hein AM, O'Banion MK (2009) Neuroinflammation and memory: the role of prostaglandins. Mol Neurobiol 40:15-32. doi:10.1007/s12035-009-8066-z

13. Salmon JA, Higgs GA (1987) Prostaglandins and leukotrienes as inflammatory mediators. Br Med Bull 43:285-296

14. Shohami E, Cohen-Yeshurun A, Magid L, Algali M, Mechoulam R (2011) Endocannabinoids and traumatic brain injury. Br J Pharmacol 163:1402-1410. doi:10.1111/j.1476-5381.2011.01343.x

15. Chen $C$ (2016) Endocannabinoid metabolism in neurodegenerative diseases. Neuroimmunol Neuroinflamm 3:268-270. doi:10.20517/2347-8659.2016.46

16. Chen R, Zhang J, Wu Y, Wang D, Feng G, Tang YP, Teng Z, Chen C (2012) Monoacylglycerol lipase is a therapeutic target for Alzheimer's disease. Cell Rep 2:1329-1339. doi:10.1016/j.celrep.2012.09.030

17. Piro JR, Benjamin DI, Duerr JM, Pi Y, Gonzales C, Wood KM, Schwartz JW, Nomura DK, Samad TA (2012) A dysregulated endocannabinoid-eicosanoid network supports pathogenesis in a mouse model of Alzheimer's disease. Cell Rep 1:617-623. doi:10.1016/j.celrep.2012.05.001 
18. Pihlaja R, Takkinen J, Eskola O, Vasara J, Lopez-Picon FR, Haaparanta-Solin M, Rinne JO (2015) Monoacylglycerol lipase inhibitor JZL184 reduces neuroinflammatory response in APdE9 mice and in adult mouse glial cells. J Neuroinflammation 12:81. doi:10.1186/s12974-015-0305-9

19. Zhang J, Hu M, Teng Z, Tang YP, Chen C (2014) Synaptic and cognitive improvements by inhibition of 2-AG metabolism are through upregulation of microRNA-188-3p in a mouse model of Alzheimer's disease. J Neurosci 34:14919-14933. doi:10.1523/jneurosci.1165-14.2014

20. Bloom GS (2014) Amyloid- $\beta$ and tau: the trigger and bullet in Alzheimer disease pathogenesis. JAMA Neurol 71:505-508. doi:10.1001/jamaneurol.2013.5847

21. Rapoport M, Dawson HN, Binder LI, Vitek MP, Ferreira A (2002) Tau is essential to beta -amyloidinduced neurotoxicity. Proc Natl Acad Sci U S A 99:6364-6369. doi:10.1073/pnas.092136199

22. Yoshiyama Y, Higuchi M, Zhang B, Huang SM, Iwata N, Saido TC, Maeda J, Suhara T, Trojanowski JQ, Lee VM (2007) Synapse loss and microglial activation precede tangles in a P301S tauopathy mouse model. Neuron 53:337-351. doi:10.1016/j.neuron.2007.01.010

23. Takashima A (2006) GSK-3 is essential in the pathogenesis of Alzheimer's disease. J Alzheimers Dis 9:309-317. doi:10.3233/jad-2006-9s335

24. Turab Naqvi AA, Hasan GM, Hassan MI (2020) Targeting Tau Hyperphosphorylation via Kinase Inhibition: Strategy to Address Alzheimer's Disease. Curr Top Med Chem 20:1059-1073. doi:10.2174/1568026620666200106125910

25. Patrick GN, Zukerberg L, Nikolic M, de la Monte S, Dikkes P, Tsai LH (1999) Conversion of p35 to p25 deregulates Cdk5 activity and promotes neurodegeneration. Nature 402:615-622. doi:10.1038/45159

26. Kimura T, Ishiguro K, Hisanaga S (2014) Physiological and pathological phosphorylation of tau by Cdk5. Front Mol Neurosci 7:65. doi:10.3389/fnmol.2014.00065

27. Zhang J, Chen C (2018) Alleviation of Neuropathology by Inhibition of Monoacylglycerol Lipase in APP Transgenic Mice Lacking CB2 Receptors. Mol Neurobiol 55:4802-4810. doi:10.1007/s12035017-0689-x

28. Chen R, Zhang J, Fan N, Teng ZQ, Wu Y, Yang H, Tang YP, Sun H, Song Y, Chen C (2013) Delta9-THCcaused synaptic and memory impairments are mediated through COX-2 signaling. Cell 155:11541165. doi:10.1016/j.cell.2013.10.042

29. Song Y, Hu M, Zhang J, Teng ZQ, Chen C (2019) A novel mechanism of synaptic and cognitive impairments mediated via microRNA-30b in Alzheimer's disease. EBioMedicine 39:409-421. doi:10.1016/j.ebiom.2018.11.059

30. Zhang J, Teng Z, Song Y, Hu M, Chen C (2015) Inhibition of monoacylglycerol lipase prevents chronic traumatic encephalopathy-like neuropathology in a mouse model of repetitive mild closed head injury. J Cereb Blood Flow Metab 35:443-453. doi:10.1038/jcbfm.2014.216

31. Selkoe DJ (2002) Alzheimer's disease is a synaptic failure. Science 298:789-791. doi:10.1126/science.1074069 
32. Bensinger SJ, Tontonoz $P$ (2008) Integration of metabolism and inflammation by lipid-activated nuclear receptors. Nature 454:470-477. doi:10.1038/nature07202

33. Villapol S (2018) Roles of Peroxisome Proliferator-Activated Receptor Gamma on Brain and Peripheral Inflammation. Cell Mol Neurobiol 38:121-132. doi:10.1007/s10571-017-0554-5

34. Long JZ, Li W, Booker L, Burston JJ, Kinsey SG, Schlosburg JE, Pavon FJ, Serrano AM, Selley DE, Parsons LH, Lichtman AH, Cravatt BF (2009) Selective blockade of 2-arachidonoylglycerol hydrolysis produces cannabinoid behavioral effects. Nat Chem Biol 5:37-44. doi:10.1038/nchembio.129

35. Long JZ, Nomura DK, Cravatt BF (2009) Characterization of monoacylglycerol lipase inhibition reveals differences in central and peripheral endocannabinoid metabolism. Chem Biol 16:744-753. doi:10.1016/j.chembiol.2009.05.009

36. Chen C (2015) Homeostatic regulation of brain functions by endocannabinoid signaling. Neural Regen Res 10:691-692. doi:10.4103/1673-5374.156947

37. Song Y, Zhang J, Chen C (2015) Fine-tuning of synaptic upscaling at excitatory synapses by endocannabinoid signaling is mediated via the CB1 receptor. Sci Rep 5:16257. doi:10.1038/srep16257

38. Ittner LM, Ke YD, Delerue F, Bi M, Gladbach A, van Eersel J, Wölfıng H, Chieng BC, Christie MJ, Napier IA, Eckert A, Staufenbiel M, Hardeman E, Götz J (2010) Dendritic function of tau mediates amyloidbeta toxicity in Alzheimer's disease mouse models. Cell 142:387-397.

doi:10.1016/j.cell.2010.06.036

39. Shipton OA, Leitz JR, Dworzak J, Acton CE, Tunbridge EM, Denk F, Dawson HN, Vitek MP, WadeMartins R, Paulsen O, Vargas-Caballero M (2011) Tau protein is required for amyloid \{beta\}-induced impairment of hippocampal long-term potentiation. J Neurosci 31:1688-1692. doi:10.1523/jneurosci.2610-10.2011

\section{Figures}



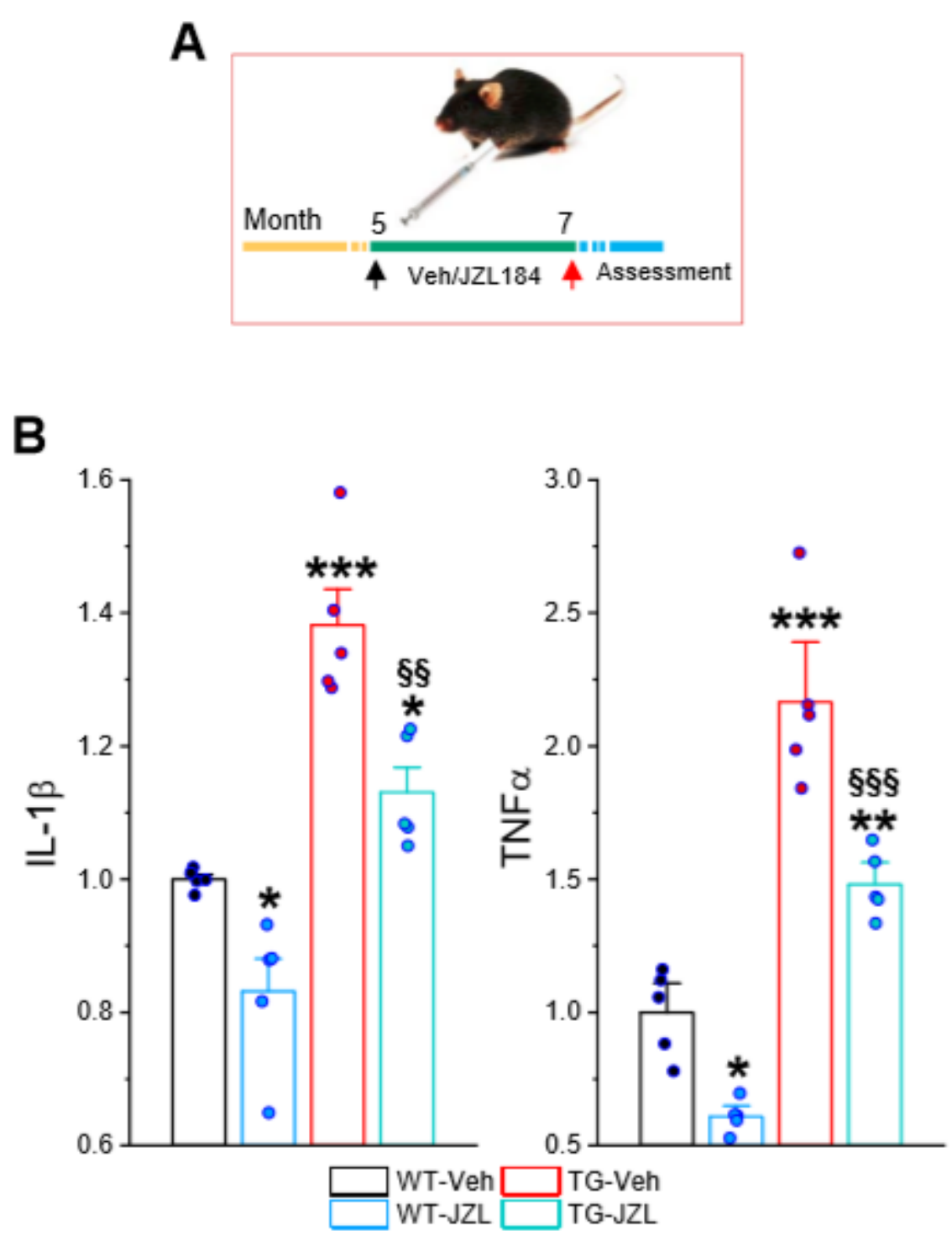

\section{Figure 1}

Inactivation of MAGL reduces proinflammatory cytokines in P301S/PS19 transgenic (TG) mice. A) Schematic illustration of the experimental protocol. PS19 TG mice and their age-matched wild-type (WT) controls received vehicle or JZL184 (10 mg/kg, i.p.) three times per week starting at 5 months of age for 8 weeks. All the assessments were made at 7 months of age. B) Changes in hippocampal IL-1 $\beta$ and TNFa in mice treated with JZL184. Cytokines were detected using ELISA analysis. Data are means \pm SEM. ${ }^{\star} \mathrm{P}<0.05,{ }^{\star} * \star \mathrm{P}<0.001$ compared with WT-vehicle; $\S \S \mathrm{P}<0.01$, $\S \S \S \mathrm{P}<0.001$ compared with TG-vehicle (ANOVA with Bonferroni test, $n=5 /$ group). 


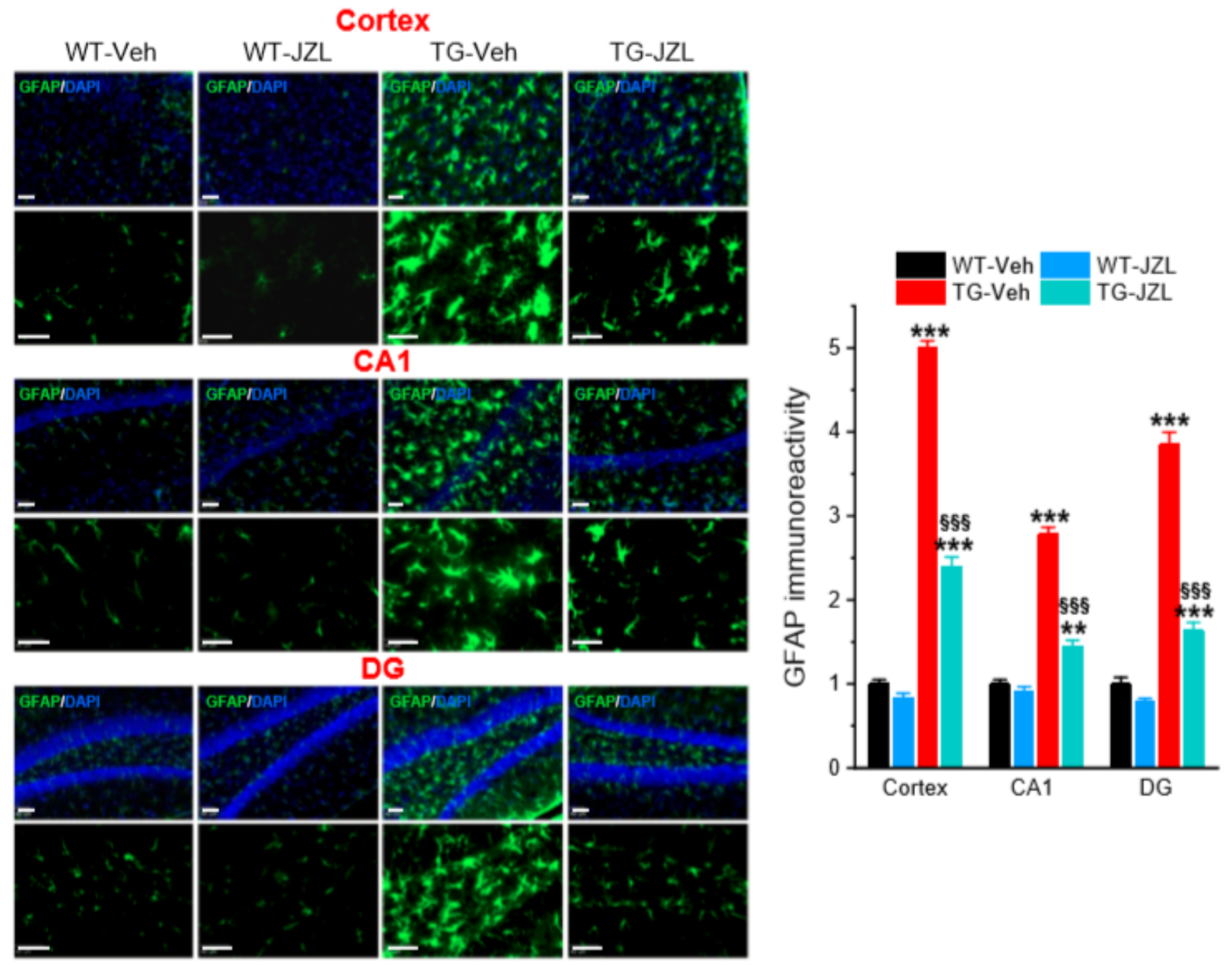

Figure 2

Inhibition of 2-AG metabolism attenuates reactivity of astrocytes in PS19 TG mice. Immunoreactivity of astrocytic marker GFAP (green) in the cortex and hippocampus is reduced in tau TG mice that received JZL184. Scale bars: $40 \mu \mathrm{m}$. Data are means $\pm S E M$. ${ }^{*}{ }^{*} P<0.01,{ }^{* \star *} \mathrm{P}<0.001$ compared with WT-vehicle control; $\S \S \S \mathrm{P}<0.001$ compared with TG-vehicle (ANOVA with Bonferroni post-hoc test, $\mathrm{n}=4$ mice per group). 


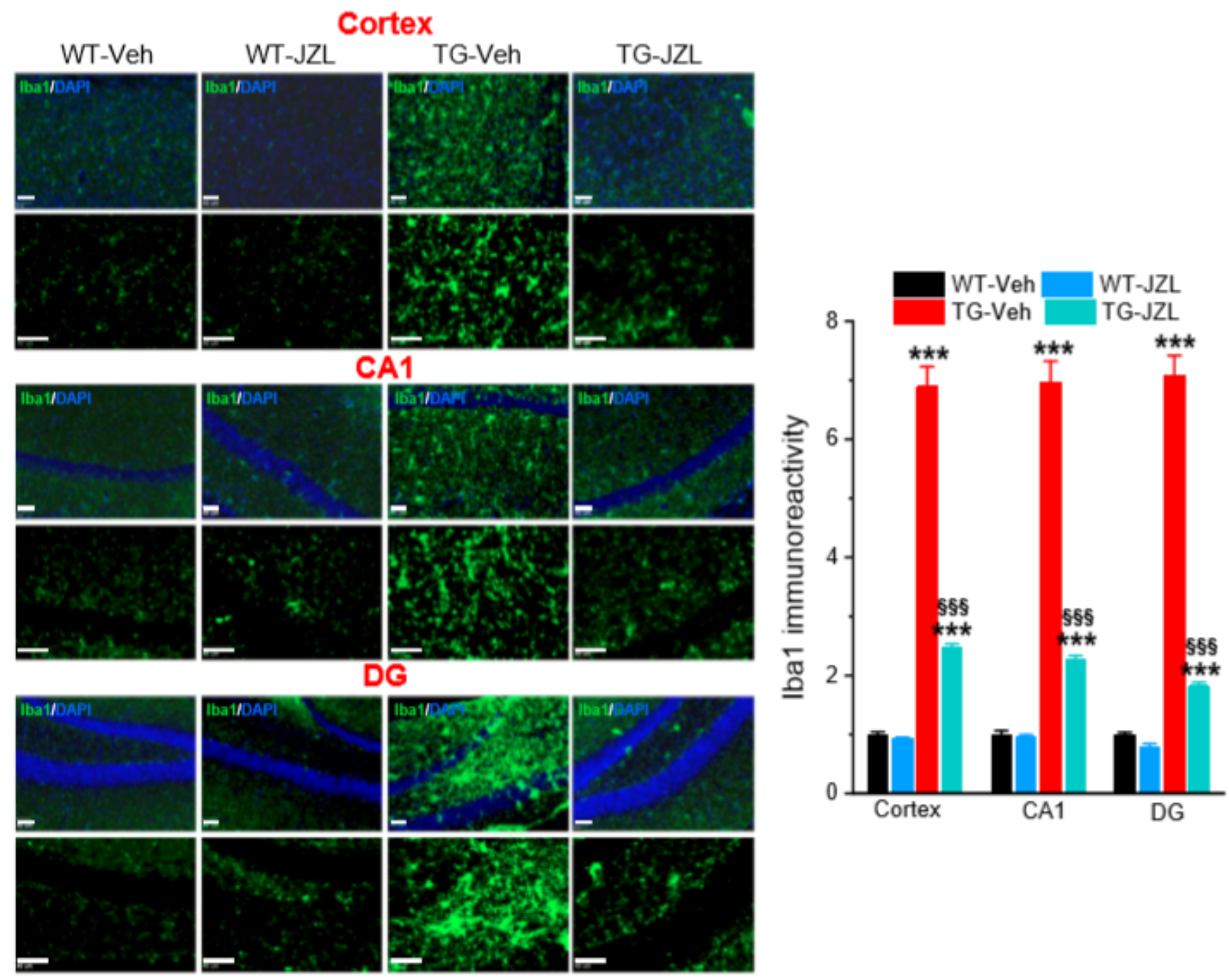

Figure 3

Inhibition of 2-AG metabolism attenuates reactivity of microglial cells in PS19 TG mice. Immunoreactivity of microglial marker Iba-1 (green) in the cortex and hippocampus is reduced in tau TG mice that received JZL184. Scale bars: $40 \mu \mathrm{m}$. Data are means \pm SEM. ${ }^{*} P<<0.01,{ }^{*} * \mathrm{P}<0.001$ compared with WT-vehicle control; $\S \S \S P<0.001$ compared with TG-vehicle (ANOVA with Bonferroni post-hoc test, $n=4$ mice per group). 

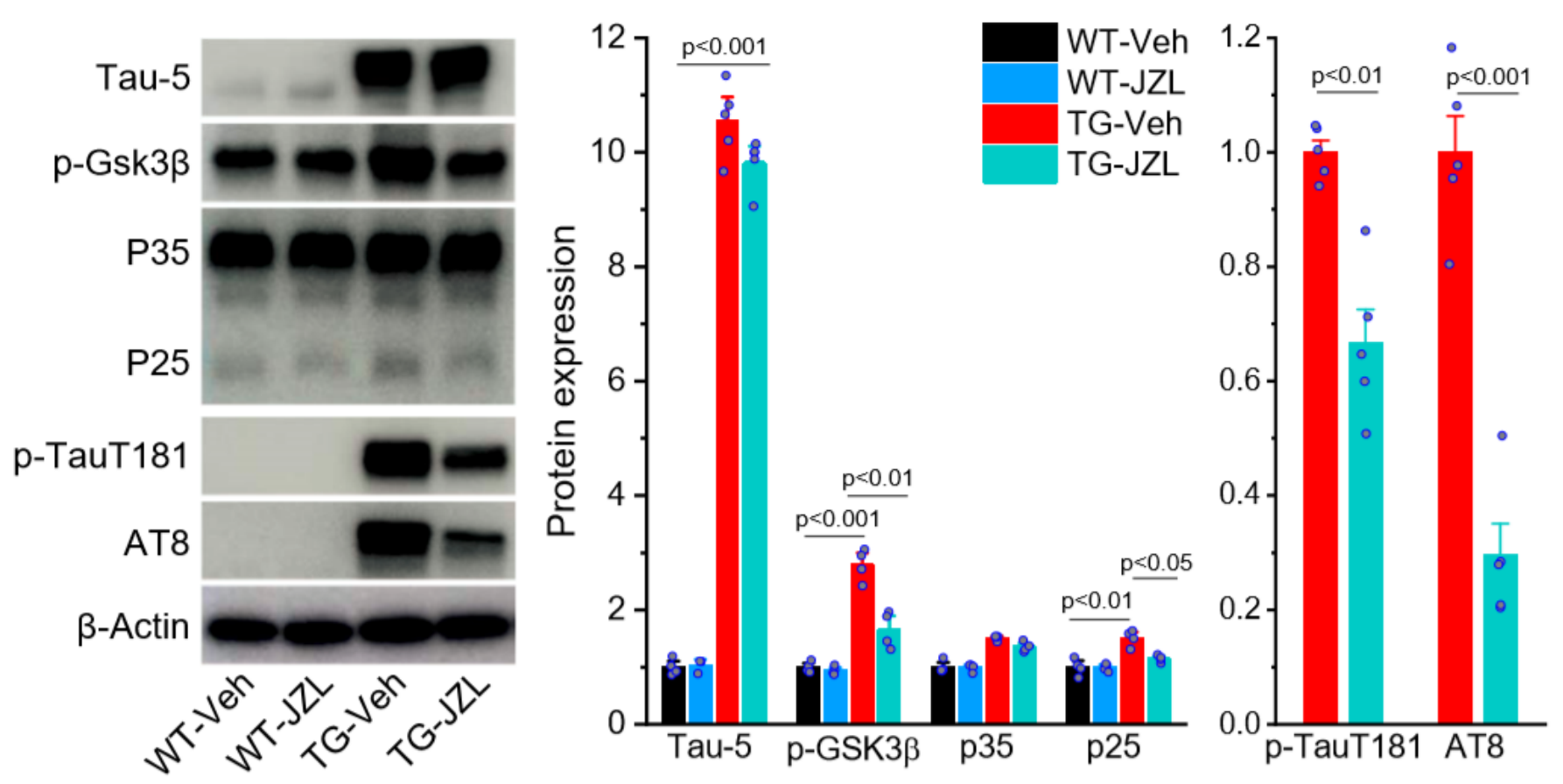

\section{Figure 4}

Inactivation of MAGL alleviates tauopathies in PS19 TG mice. Immunoblot analysis of phosphorylated tau in PS19 TG mice that received JZL184 for 8 weeks. Tau-5 (total tau), p-tauT181, p-tau Ser202/Thr205 (AT8), GSK3 $\beta, P 35 / 25$ in the hippocampus were assessed. The values of Tau-5, P35/25 and GSK3 $\beta$ are normalized to WT-vehicle, while p-tauT181 and AT8 are normalized to TG-vehicle as these phosphorylated tau proteins are not detectable in WT mice. Data are means \pm SEM (ANOVA with Fisher PLSD test, $\mathrm{n}=4 \sim 5 /$ group). 
A

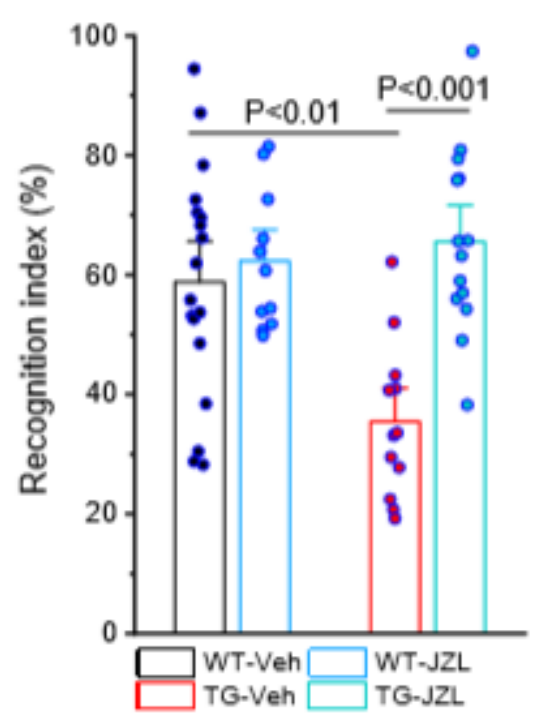

C

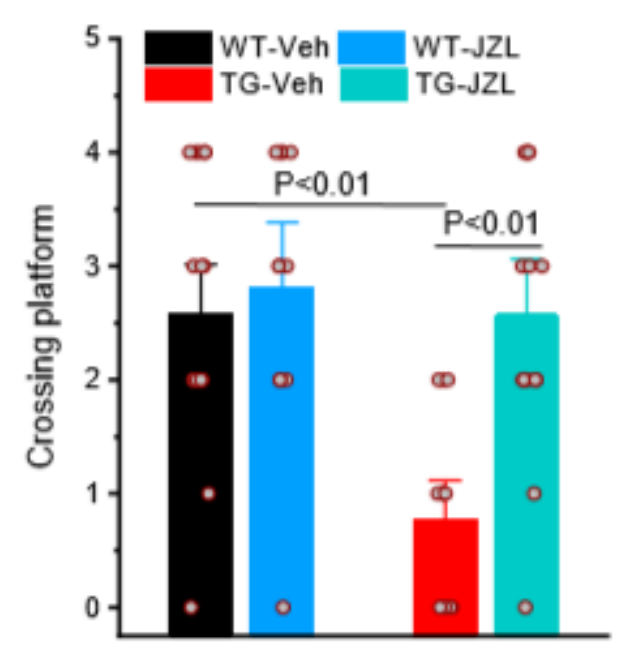

B
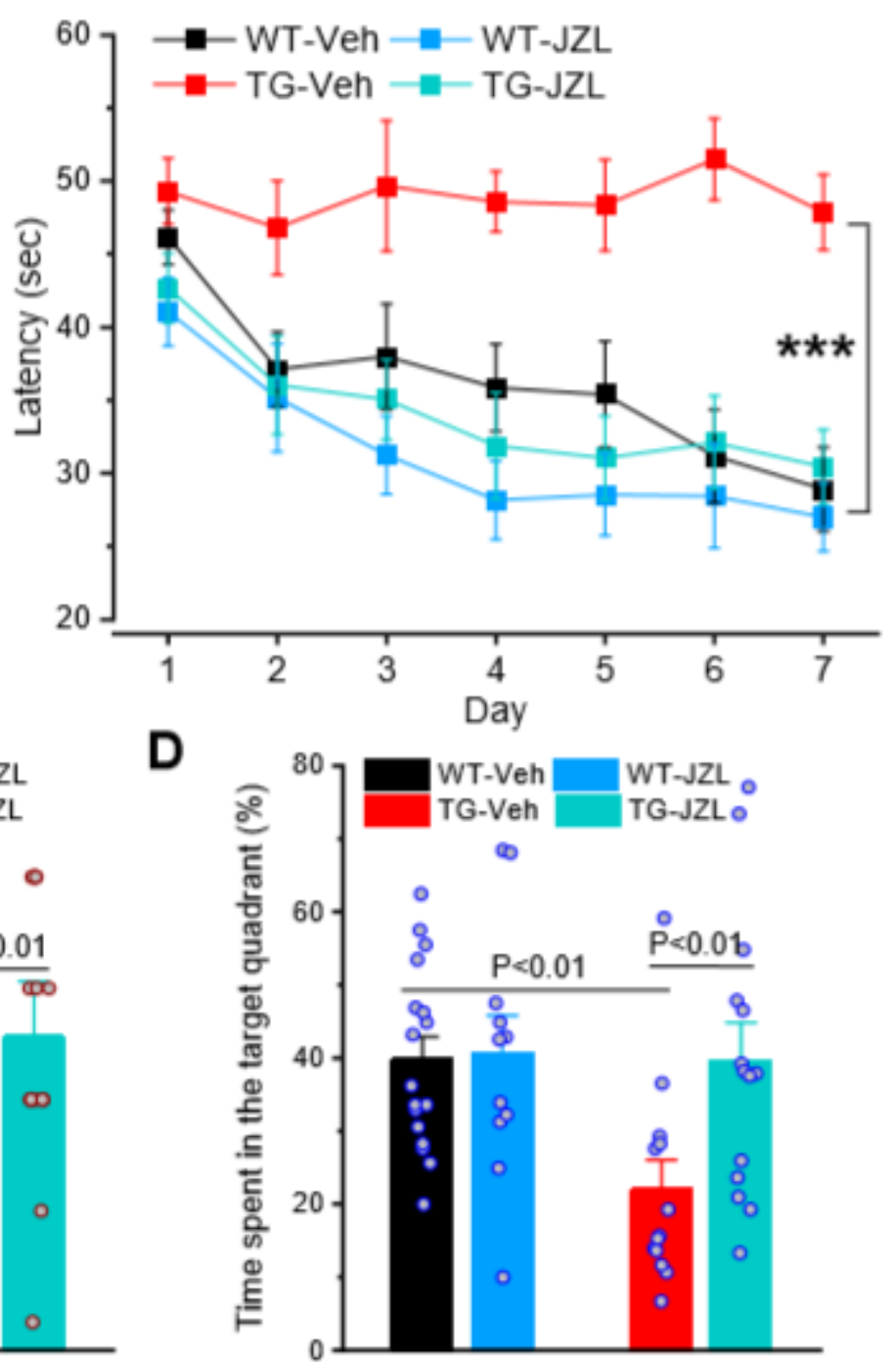

\section{Figure 5}

Inhibition of MAGL improves cognitive function in PS19 TG mice. A) The novel object recognition test in 7-month-old PS19 TG mice that received JZL184 for 8 weeks. Data are means \pm SEM (ANOVA with Bonferroni post-hoc test, $\mathrm{n}=11 \sim 18 /$ group). B) The Morris water maze test was performed in 7-month-old PS19 TG mice that received JZL184 for 8 weeks. Data are means $\pm S E M(* \star * P<0.001$, ANOVA with repeated measures, $n=11 \sim 17 /$ group). C) Number of platform crossing during the probe test. The probe trial was conducted $24 \mathrm{hrs}$ after 7 days of acquisition training. Data are means $\pm S E M$ (ANOVA with Bonferroni post-hoc test, $n=11 \sim 17 /$ group). D) The percentage of time spent in search of the target quadrant during the probe trial. Data are means \pm SEM (ANOVA with Bonferroni post-hoc test, $\mathrm{n}=11 \sim 17 /$ group). 


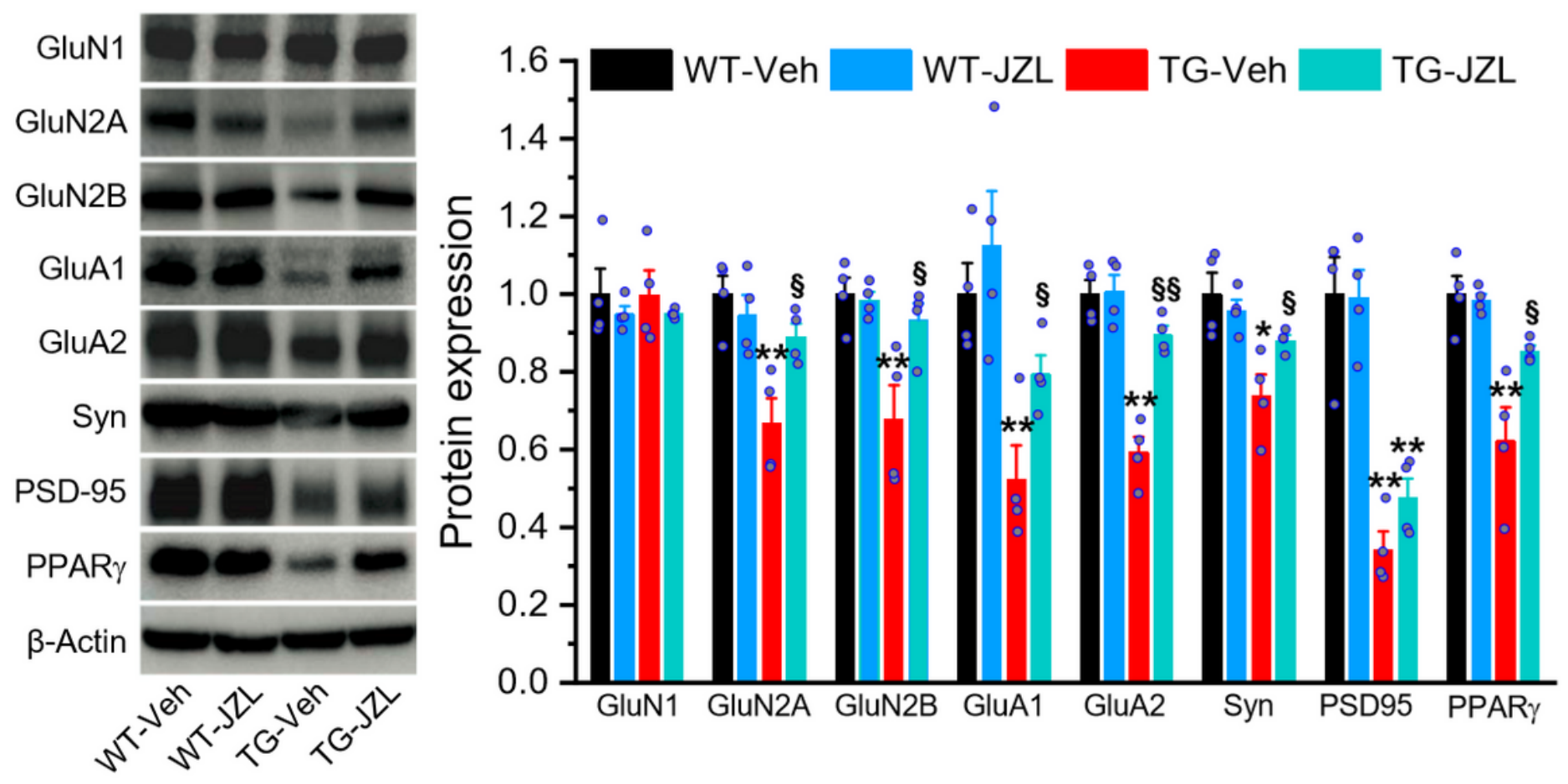

Figure 6

Inactivation of MAGL prevents deterioration in expression of synaptic proteins in PS19 TG mice. Immunoblot analysis of hippocampal expression of AMPA (GluA1 and GluA2), and NMDA (GluN1, GluN2A, and GluN2B) receptor subunits, PSD-95, synaptophysin (Syn), and PPARy in 7- month-old PS19 TG mice treated with vehicle or JZL184. Data are means \pm SEM. ${ }^{*} P<0.05 ; * \star P<0.01$ compared with the WTvehicle, $\S \mathrm{P}<0.05, \S \S \mathrm{P}<0.01$ compared with the TG-vehicle (ANOVA with Fisher's PLSD test, $\mathrm{n}=4$ mice per group). 